\title{
Eficiência de braquetes convencionais e autoligados considerando seu atrito e expressão de torque durante o tratamento
}

\author{
Efficiency of conventional and self-connected brackets considering its friction and torque \\ expression during treatment
}

\author{
Júlia Jordão", Marcos Valério Ferrari ${ }^{\ominus}$, Valdinéia Maria Tognetti ${ }^{\bullet}$ \\ Universidade São Francisco, Bragança Paulista, São Paulo, Brasil. *Autor para correspondência. E-mail: juliajordao_@hotmail.com
}

\begin{abstract}
Resumo: Introdução: Braquetes convencionais e autoligados comumente são avaliados em relação ao seu diferencial de eficiência na ortodontia, levando a compreensão que braquetes autoligados possuem menor atrito, devido sua ausência de ligaduras elásticas, possibilitando melhor deslizamento, enquanto os braquetes convencionais com ligaduras elásticas proporcionam maior atrito. Compara-se os níveis de eficiência entre os modelos de braquetes presentes no mercado, avaliando além do atrito, sua expressão de torque durante o tratamento. Revisão e discussão: O consequente trabalho propôs a realizar uma revisão de literatura acerca do assunto, analisando o diferencial de eficiência entre os modelos de braquetes convencionais e autoligados. Considerações finais: Concluindo-se através dos artigos científicos referenciados que não existem evidências suficientes da superioridade de eficiência dos braquetes autoligados em relação aos convencionais, mas sim, diferenciais durante o tratamento.
\end{abstract}

Palavras-chave: atrito, braquetes ortodônticos, eficiência, torque.

\begin{abstract}
Introduction: Conventional and self-ligating brackets are commonly evaluated in relation to their efficiency differential in orthodontics, leading to the understanding that self-ligating brackets have less friction, due to their absence of elastic bandages, allowing for better sliding, while conventional brackets with elastic bandages provide greater friction. The efficiency levels are compared between the models of brackets on the market, evaluating in addition to friction, their torque expression during treatment. Review and discussion: The consequent study proposed to implement a literature review on the subject, analyzing the efficiency differential between the models of conventional and self-ligating brackets. Final considerations: In conclusion, through the referenced scientific articles, there is insufficient evidence of the superior efficiency of self-ligating brackets in relation to conventional brackets, but rather, differentials during treatment.
\end{abstract}

Keywords: friction, orthodontic brackets, efficiency, torque.

\section{Introdução}

Não se sabe ao certo quando o ser humano esteve disposto a corrigir ou alinhar a dentição, contudo o tratamento e correção dos problemas oclusais, desordens da articulação temporomandibular e displasias de crescimento são partes integrantes da ortodontia, uma especialidade da odontologia, conforme explicava Novais (2014).

Segundo Vilella (2007) a ortodontia é dentro da odontologia a mais antiga das especialidades a se organizar. A correção de dentes mal posicionados são desejos que existem desde a antiguidade, pelo menos 1000 anos a.C. a busca pelo conhecimento das posições dentárias são descritos em escavações e relatos. Pierre Fauchard, conhecido como o "pai da odontologia moderna", apresentou um aparelho designado bandeau, formado por uma tira de metal em formato de arco que possuía perfurações em locais apropriados.

Edward H. Angle, no final do século XIX iniciou os conceitos e a importância da oclusão, esquematizou a Classificação de Angle para as maloclusões, utilizada até os dias de hoje, sendo considerado o "pai" da ortodontia moderna. Angle apresentava a importância da análise facial para realizar diagnóstico e planejamento do tratamento ortodôntico, percebendo que uma oclusão harmônica e funcional também seria o objetivo da ortodontia, além do alinhamento dos dentes, conforme Novais (2014). 
Os braquetes são ferramentas habituais no tratamento ortodôntico. Para Eberting et al. (2001) a velocidade em que os dentes irão se movimentar e por assim a duração que terá o tratamento são determinados pelo tipo de fio ou ligadura e tipo de braquete.

Atualmente utilizam-se dois tipos de braquetes, o convencional e o autoligado. Mais comumente utilizados são os braquetes convencionais com ligaduras elásticas ou fios de aço inoxidável, enquanto, segundo Juneja et al. (2014), os braquetes autoligados não possuem ligaduras, e sim, um dispositivo mecânico de clipe ativo ou slide passivo, presente no braquete, capaz de fechar a sua canaleta. O braquete autoligado foi trazido ao mercado para permitir uma mecânica de deslizamento melhor, pois um ambiente "livre de fricção" seria criado, permitindo que o tempo de tratamento diminuísse, pois os dentes se moveriam de maneira mais rápida.

Conforme Satlher et al. (2011) foi na década de 30 que o conceito de braquetes sem ligaduras nasceu com o aparelho de Russell Lock, na tentativa de obter eficiência clínica junto com um tempo reduzido com a ligação dos braquetes.

Devido ao seu maior uso e valor mais acessível, os braquetes convencionais com ligaduras elásticas estão mais presentes nos consultórios odontológicos, no entanto vem sendo discutido sobre seu uso, considerando os braquetes autoligados mais eficientes, capazes de reduzir, segundo Castro (2009), em até quatro vezes o tempo de trabalho comparando-os aos convencionais. Além da redução do tempo de tratamento, a classificação dos autoligados serem responsáveis por causarem menor atrito também já consistem em pesquisas e conclusões que a ligadura elástica é responsável por $50 \%$ de atrito, além de aumentarem o nível de dor do paciente e proporcionarem maior acúmulo de biofilme e consequentemente prejuízo à saúde periodontal, de acordo com Satlher et al. (2011).

Conforme Maltagliati (2010) os braquetes autoligados ativos e passivos apresentam conversão da canaleta em tubo, pois a quarta parede móvel é a responsável por isso, sendo assim os braquetes autoligados passivos possuem a canaleta fechada por uma trava externa, transformando-o em tubo, enquanto nos braquetes ativos, o fechamento é feito por um clipe que passa por uma parte da parede superior ou inferior da canaleta.

Para Morina et al. (2008) é um grande desafio a introdução dos braquetes autoligados ativos e passivos, devido ao diferente modo de ligadura e as grandes alterações na expressão de carga durante a mecanoterapia, pois alguns desses sistemas apresentam menor atrito em vitro e características de torção desconhecidas.

De acordo com Turpin (2009), citado por Satlher et al. (2011) os estudos dos aparelhos autoligados feitos em modelos, obtendo resultados in vitro devem ser analisados com cuidado, pelo fato desses não corresponderem à realidade absoluta por falta dos tecidos duros e moles do paciente.

De acordo com Morina et al. (2008) o torque utilizado na ortodontia, responsável por alterar a inclinação dos dentes, mudanças vestíbulo-lingual depende do tipo de braquete, da rigidez do torque do fio, o espaço do fio/ranhura e o modo de ligadura empregado.

Por conseguinte, para Castro (2009) não existem evidências suficientes da superioridade de eficiência dos braquetes autoligados em relação aos convencionais, é necessário analisar cada paciente para a escolha ideal de qual braquete para o tratamento ortodôntico, além da necessidade de mais estudos sobre a comparação de eficiência.

O presente trabalho teve por justificativa e objetivo apresentar uma revisão de literatura dos modelos de braquetes convencionais e autoligados, considerando suas apresentações e comparando seu diferencial de eficiência, analisando suas forças de atrito e expressão de torque durante o tratamento.

\section{Revisão}

Presentes conceitualmente na ortodontia por Russell Lock desde a década de 30, os braquetes autoligados tentam alcançar uma eficiência de redução de tempo de tratamento gasto sem o uso de ligaduras, conforme Satlher et al. (2011).

Atualmente, de acordo com Satlher et al. (2011) a comparação de eficiência e tempo de trabalho reduzido entre braquetes autoligados e convencionais vem aumentando, considerando os braquetes autoligados um sistema de tratamento sem ligaduras elásticas capazes de reduzir o atrito durante as mecânicas de deslize que os braquetes convencionais que fazem uso de ligaduras para contenção do fio ortodôntico ainda não são capazes. Sua classificação se divide em três formas: ativos, quando o fio é pressionado dentro da canaleta, passivos, quando existe a liberdade do fio na canaleta, ou interativos, quando realizado ambas as funções anteriores, dependendo da espessura e calibre do fio.

De acordo com Tweeny \& Hughes (1961) citado por Pizzoni et al. (1998) o atrito pode ser definido como a resistência ao movimento quando tenta deslizar uma superfície sobre a outra, estando estas em contato. Para 
Didática Ortodôntica, Atrito em Ortodontia, Youtube (2018) o atrito na ortodontia existe quando há deslizamento de superfícies em contato e nessa surge uma força na direção oposta a esse deslizamento. É necessárias duas forças de atrito, o atrito estático e o atrito cinético, o primeiro é a menor força necessária para que ocorra o início de um movimento, enquanto o cinético é a resistência que se deve ter quando um objeto sólido se movimenta sobre outro em uma velocidade constante. Para compensar o atrito é importante tomar cuidado como o excesso de força e limitá-lo, pois pode ocorrer desconforto, reabsorção radicular e perda de ancoragem no paciente. A movimentação do dente depende da força empregada considerando o atrito presente entre o braquete e o fio ortodôntico, assim ambos possuindo um coeficiente de atrito menor é o ideal. Braquetes com ligaduras elásticas, como é o caso dos braquetes convencionais causam uma variação no nível de força, pois causam um aumento na resistência friccional, definida como uma força contrária ao movimento ortodôntico, concluindo-se que os braquetes autoligados causam menores forças de atrito quando comparados. Entretanto o atrito possui finalidade de controlar as inclinações, rotações e angulações, compreendendo que devido a ele uma movimentação desejável possa ser conferida quando necessitamos de um travamento entre a canaleta do braquete e a ligadura para realizar alguma movimentação.

Segundo Faria (2010) a grande preocupação com o atrito em ortodontia se deu pelo uso frequente das mecânicas de deslizamento, que buscam o sucesso do tratamento. Para Pizzoni et al. (1998) um braquete carregado por rolamentos de esferas lineares de baixa fricção tem por função deslizar ao longo de um arco esticado, tendo um atrito conhecido e mínimo tanto em ângulo com o fio, quanto paralelo.

Dentre tantos estudos, destaca-se o feito por Voudouris, que mediu o atrito produzido por três tipos de braquetes convencionais e os comparou com três tipos de braquetes autoligáveis: um ativo (Sigma) e dois passivos (Damon SL e Interactwin). Quando arcos 0,019"x0,025" de aço foram encaixados, um braquete convencional com ligadura em "O" produziu atrito entre 371 vezes e 667,8 vezes maior do que os braquetes autoligáveis passivos. Os braquetes convencionais com ligaduras metálicas apresentaram valores de atrito até 532,8 vezes maior em relação aos braquetes autoligáveis passivos. Os autoligáveis ativos produziram até 310 vezes maior atrito do que os autoligáveis passivos (Satlher et al., 2011).

Conforme Geremia et al. (2015) braquetes autoligados passivos e ativos têm a quarta parede móvel, responsável por converter a canaleta em tudo, tendo como principais vantagens: diminuição do atrito, capacidade de melhorar a mecânica de deslize; menor assistência ao lado da cadeira odontológica e redução do tempo de cadeira, pois a colocação e retirada do arco são mais rápidas.

Para Geremia et al. (2015) os relatos de atrito reduzido dos braquetes autoligados ainda são controversos quando são comparados com os braquetes convencionais. O fato de os estudos serem realizados de forma in vitro podem diferenciar com os estudos clínicos, pois outros meios interferem como o próprio movimento do dente, já que este estará fixado a um braquete responsável pelo deslize, segundo Geremia et al. (2015).

Para obter uma correta inclinação vestíbulo-lingual dos dentes, o torque se faz necessário no tratamento. Para Franco et al. (2015) o torque é a ocasião gerada pela torção de um fio retangular na ranhura de suporte.

De acordo com Franco et al. (2015) existem muitos fatores capazes de influenciar e alterar durante o tratamento ortodôntico o torque, sendo eles: espessura do fio; tamanho da canaleta; magnitude da torção; posicionamento do braquete e do dente; composição do braquete e do fio; profundidade e largura do fio; diferença das ligas constituintes dos fios; processo de fabricação dos suportes; tolerância de fabricação de suportes e fios.

Para compreender o mecanismo de fechamento dos braquetes autoligados que serão necessários para realização da expressão de torque, Thomali et al. (2017) explica que o braquete possui a capacidade de se prender ao arco, pois possuem um dispositivo para fechamento da canaleta, nos braquetes ativos esse clipe exerce pressão sobre o fio do arco, enquanto no braquete passivo o clipe é responsável por transformar a canaleta aberta em tubo.

Conforme Franco et al. (2015) as características de torque não foram estudadas em detalhes ainda, pois é de alta complexidade e existem poucos estudos que avaliam a expressão de torque. Realizou, portanto, um estudo in vitro para comparar a expressão de torque em braquetes autoligados passivos e ativos e braquetes convencionais, em diferentes ângulos de torção do fio. Foram utilizados 30 braquetes de incisivos centrais superiores de seis marcas, sendo cinco autoligados tanto ativos quanto passivos e um braquete convencional com ligadura de elastômero. $\mathrm{O}$ tamanho da canaleta utilizado foi de $0,022 \times 0,028$ pol. Os fios foram de aço inoxidável da Morelli, Sorocaba, SP, Brasil de seção retangular de 0,019 x 0,025 pol, com 3,5 cm de comprimento. 
Para que os braquetes pudessem ser colados, Franco et al. (2015) explica que cilindros metálicos de aço inoxidável de $4 \mathrm{~cm}$ de comprimento e aproximadamente $1 \mathrm{~cm}$ de diâmetro foram fabricados. A linha de base para realização dos testes foi a posição de torque zero e foi utilizado uma máquina universal de ensaios EMIC DL2000 (equipamento científico Instron Brasil - Paraná - Brasil) para realizar a torção do fio simetricamente em ambas as extremidades. Para não ocorrer torques e forças residuais, a cada teste, a reinicialização e nivelamento da máquina no sistema era realizado.

Franco et al. (2015) explica que foi utilizado um transdutor para medida de torque e o sensor nele contido captava a força, torques e ângulos de torção aplicados e assim eram transferidos eletronicamente para o computador em forma de dados. Foram feitos testes em torções de $12^{\circ}, 24^{\circ}, 36^{\circ}$ e $48^{\circ}$ e cada torção foi testada dez vezes a combinação de braquete/fio, avaliados em Nmm.

Para obter bons resultados nos tratamentos ortodônticos, o torque é de grande importância, Franco et al. (2015) complementa que sua precisão está relacionada diretamente aos resultados que são desejados na oclusão e estética do tratamento.

Segundo Franco et al. (2015) a eficácia do torque é influenciada pelo tamanho e conformação da canaleta, como as fendas dos braquetes, que segundo ele estão acima do tamanho informado pelo fabricante, causaram uma folga maior entre a canaleta e o fio prejudicando na mecânica do torque expresso, pois essa relação é considerada mais importante do que sistemas de ligadura das diferentes marcas de braquetes. Imagina-se que a expressão inicial de torque depende da profundidade da canaleta, ou seja, o fio quando entra em contato com o sistema de conexão já é iniciado uma expressão de torque, mesmo o fio não tendo encontrado as paredes cervicais e incisais da canaleta. No entanto, não é possível realizar movimentos ortodônticos efetivos com esse torque precoce, pois a eficácia clínica só acontece quando o fio encosta nas paredes cervicais e incisais.

Franco et al. (2015) ainda mostrou que o braquete autoligado passivo, anteriormente mostrou um ângulo de torção de $7,93^{\circ}$ e $5 \mathrm{Nmm}$ obtendo capacidade de expressar um torque clinicamente eficaz. Na literatura, é considerado clinicamente eficazes momentos de forças que variam de 5 a $20 \mathrm{Nmm}$. Enquanto o braquete autoligado ativo apresentou uma torção de $21,4^{\circ}$ para chegar nos $5 \mathrm{Nmm}$, exibiu um comportamento que seria necessário torções maiores no fio, variando de $21,4^{\circ}$ a $39,01^{\circ}$ para ser clinicamente factível.

As análises e conclusões do presente estudo in vitro realizada por Franco et al. (2015) foi relatado que houve diferenças em todas as torções estudadas e o braquete autoligado ativo obteve sua menor expressão de torque em todas as torções que foram testadas, enquanto o braquete autoligado passivo obteve o torque mais alto até o $36^{\circ}$, pois no $48^{\circ}$ outros dois braquetes, sendo um ativo e outro passivo se assemelharam com momentos de torque do braquete passivo. O braquete convencional ficou em terceiro em melhor expressão de torque do estudo avaliado. Quando foi testado a faixa de torque considerada clinicamente eficaz, o braquete passivo foi o primeiro a expressar o torque clinicamente eficaz e o braquete autoligado ativo foi o último a expressar um torque clinicamente eficaz.

Franco et al. (2015) terminou concluindo que a conexão fio e braquete tanto de braquetes autoligados ativos e passivos ou convencionais com ligadura elástica semelha não intervir na expressão final de torque, sendo o convencional dependente da interação do fio e do braquete escolhido para utilizar-se da mecânica ortodôntica.

Diante disso, Kumar et al. (2016) expõe que apesar de apresentar a vantagem de não necessitar de visitas frequentes ao ortodontista para troca de ligadura e proporcionar um encaixe consistente do fio, os braquetes autoligados além do alto custo, apresentam problemas na expressão de torque completa do braquete, seu manejo é bem mais complicado, apresentando mais falhas do que os convencionais.

\section{Discussão}

De acordo com Vilella (2007) a ortodontia no Brasil passou por evolução desde que chegou como uma nova ciência até os dias que tornaram cursos de doutorado. Para Novais (2014) não se sabe historicamente quando as pessoas decidiram corrigir os dentes.

Quando o braquete não possui as ligaduras, como é o caso do braquete autoligado é proporcionado uma melhor mecânica de deslizamento, pois o tratamento é realizado em um ambiente livre de atrito, capaz de uma eficácia em menor tempo de tratamento, conforme Eberting et al. (2001), Juneja et al. (2014) e Satlher et al. (2011).

Segundo Morais (2012) os braquetes autoligados possuem defensores que acreditam na sua superioridade quando relacionados aos braquetes convencionais e quando comparados aos braquetes autoligados ativos, os 
braquetes autoligados passivos também tem sua superioridade, entretanto os defensores de braquetes autoligados ativos defendem que sua eficácia clínica é melhor. No entanto, os braquetes autoligados tem preço superior aos convencionais, sendo importante a necessidade de conhecimento das propriedades de cada aparelho. Para Kumar et al. (2016) além dos braquetes autoligados apresentarem um custo maior, seu uso é mais complicado e mais apto a exibir falhas. Castro (2009) sugeriu novos estudos, sendo eles clínicos para que se possa abordar a comparação entre a mecânica dos braquetes autoligados e convencionais, principalmente a estabilidade em tratamentos com os braquetes autoligados.

Para a movimentação ortodôntica, as forças aplicadas devem superar o atrito tanto o cinético como o estático, sendo este dependente da interação entre a canaleta de um braquete ou de uma ligadura e de um fio ortodôntico, conforme explica Geremia et al. (2015). A literatura ainda não possui estudos relacionados aos braquetes e as ligaduras de elástico, mas através dos estudos de Geremia et al. (2015) estes ainda apresentavam maior força de atrito quando comparados aos braquetes autoligados passivos.

Conforme Morais (2012) uma quantidade de força realizada deve exceder a resistência ao deslizamento para que aconteça a movimentação dos dentes. Expõe que utilizar-se do termo "resistência ao deslizamento" é o mais apropriado para descrever os termos de atrito ou friç̧ão, pois essa resistência é o que o braquete se depara quando se desliza pelo fio. Composta pela fricção clássica + binding, que seria o contato que acontece entre o fio e a borda do braquete em movimentos de angulação + notching, que seria quando o contato fio e braquete formam um ângulo de contato muito alto, causando deformação no fio.

Para Didática Ortodôntica, Atrito em Ortodontia, Youtube (2018), apesar das menores forças de atrito serem o desejável no tratamento, ele também é responsável pelo controle de movimentos como as rotações e inclinações, portanto um braquete que possui ligadura é capaz de um travamento entre a canaleta e a própria ligadura.

A necessidade de comparar o material que o braquete foi produzido é imprescindível para relacionar ao atrito, pois a rugosidade da superfície está ligada e interfere diretamente na produção da quantidade de atrito, como também observar que o modo de desalinhamento dos dentes é influência para a quantidade de atrito, segundo Jakob et al. (2014). O atrito tem grande importância na força que será aplicada nos dentes, Pizzoni et al. (1998).

Conforme Sims et al. (1993) citado por Maltagliati (2010) os modelos de braquetes autoligados passivos apresentavam superioridade em relação aos ativos, no entanto ambos se apresentavam melhor do que os braquetes convencionais quando comparados em redução de atrito.

Logo Franco et al. (2015) e Thomali et al. (2017) concluíram que para uma boa oclusão e estética do tratamento ortodôntico, o torque é parte fundamental para obtenção de resultados. Com os braquetes autoligados, a expressão de torque ainda apresenta características desconhecidas, pois o torque precisa do atrito para realização da inclinação vestíbulo-lingual, segundo Morina et al (2008).

Para Shivapuja \& Berger (1994), e Alpern (2008) citados por Morais (2012) os braquetes autoligados ativos proporcionam melhor controle da posição dos dentes. Entretanto Pandis et al. (2006) e Stefanos et al. (2010) citados por Morais (2012) uma menor resistência friccional seja o melhor na fase de alinhamento e nivelamento ou para fechar espaços através de deslizamento, mas para uma melhor expressão de torque uma maior resistência a fricção é necessária.

Devido uma menor resistência friccional que os braquetes autoligados passivos apresentam, espera-se um menor controle de torque, no entanto este apresenta resultados semelhantes aos braquetes convencionais com ligaduras metálicas ou elásticas. Quando comparados com os braquetes autoligados ativos, apresentaram uma pequena melhora na expressão de torque, mas uma diferença que não foi estatisticamente significante. No entanto, estudos mais recentes constataram que os braquetes autoligados ativos não apresentaram superioridade quando comparados aos braquetes convencionais com ligaduras elásticas ou metálicas e os braquetes autoligados passivos, segundo Brauchli et al. (2011) citado por Morais (2012) e Faria (2010).

\section{Considerações finais}

Baseado na literatura revisada percebe-se que os estudos realizados são in vitro para comparação de força de atrito e expressão de torque. $\mathrm{O}$ tipo de braquete a ser selecionado varia de acordo com cada paciente e tratamento a ser realizado, pois é parte fundamental para a escolha e determinação da duração do tratamento. Cada braquete apresenta sua vantagem em determinado momento do tratamento. 
Braquetes autoligados, em especial os braquetes autoligados passivos, apresentam menor atrito quando comparados aos braquetes convencionais, devido as ligaduras de elástico serem responsáveis por esse travamento, no entanto a expressão de torque é melhor desenvolvida em braquetes convencionais, entretanto esses resultados ainda são controversos. Percebendo-se também que os braquetes autoligados passivos assemelham-se muito aos braquetes convencionais com ligaduras.

Contudo novos estudos devem ser realizados, principalmente em meio clínico para comprovação da superioridade de eficiência desses braquetes autoligados aos convencionais.

\section{Referências}

Castro, R. 2009. Braquetes autoligados: eficiência x evidências científicas. Revista Dental Press de Ortodontia e Ortopedia Facial, 14(4), 20-24.

Didática ortodôntica. Youtube. (2018). Atrito em ortodontia. Disponível em: $<$ https://www.youtube.com/watch?v=h9g6PqgxOpY\&t=203s >. Acesso em 22 de out. 2020.

Eberting, J. J., Straja, S. R., \& Tuncay, O. C. 2001. Treatment time, outcome, and patient satisfaction comparisons of Damon and conventional brackets. Clinical Orthodontics and Research, 4(4), 228-34.

Faria, R. A. 2010. Braquetes autoligados comparados aos braquetes convencionais. Monografia (Especialização) - Curso de Ortodontoa, Instituto de Ciências da Saúde Funorte/Soebrás, Alfenas, 39p.

Franco, E. M. F., Valarelli, F. P., Fernandes, J. B., Cançado, R. H., \& Freitas, K. M. S. 2015. Comparative study of torque expression among active and passive self-ligating and conventional brackets. Revista Dental Press de Ortodontia e Ortopedia Facial, 20(6), 68-74.

Geremia, J. R., Oliveira, P. S., \& Motta, R. H. L. 2015. Comparação da força de atrito entre braquetes autoligados e braquetes convencionais com diferentes ligaduras. Ortho Science: Orthodontics science and practice, 8(29), 30-37.

Jakob, S. R., Matheus, D., Pellegrin, M. C. J., Turssi, C. P., \& Amaral, F. L. B. 2014. Comparative study of friction between metallic and conventional interactive self-ligating brackets in different alignment conditions. Revista Dental Press de Ortodontia e Ortopedia Facial, 19(3), 82-89.

Juneja, P., Shivaprakash, G., Chopra, S. S., \& Kambalyal, P. B. 2014. Comparative evaluation of anchorage loss between self-ligating appliance and Conventional pre-adjusted edgewise appliance using sliding mechanics - A retrospective study. Medical Journal Armed Forces India, 71(Suppl 2), S362-S368.

Kumar, D., Dua, V., Mangla, M., Solanki, R., Solanki, M., \& Sharma, R. 2016. Frictional force released during sliding mechanics in nonconventional elastomerics and self-ligation: An in vitro comparative study. Indian Journal of Dentistry, 7(2), 60-65.

Maltagliati, L. A. 2010. Braquetes autoligados ativos x passivos. Revista Clínica de Ortodontia Dental Press, 9(2), 8-13.

Morais, J. F. 2012. Avaliação dos efeitos do sistema Damon nas inclinações dentárias, dimensões dos arcos e suporte ósseo alveolar por meio da tomografia computadorizada de feixe cônico. Tese (Doutorado) - Curso de Odontologia, Universidade de São Paulo, Bauru, 245p.

Morina, E., Eliades, T., Pandis, N., Jäger, A., \& Bourauel, C. 2008. Torque expression of self-ligating brackets compared with conventional metallic, ceramic, and plastic brackets. The European Journal of Orthodontics, 30(3), 233-238.

Novais, A. 2014. Ortodontia - Conceito e Objetivo. In: Fundamentos de Ortodontia e Próteses. $1^{\mathrm{a}}$ ed. São Paulo: Editora Saraiva, 63-64.

Pizzoni, L., Ravnholt, G., \& Melsen, B. 1998. Frictional forces related to self-ligating brackets. The European Journal of Orthodontics, 20(3), 283-291.

Sathler, R., Silva, R. G., Janson, G. J., Branco, N. C. C., \& Zanda, M. 2011. Desmistificando os braquetes autoligáveis. Dental Press Journal of Orthodontics, 16(2), 50.e1-8.

Thomali, Y. A., Mohamed, R. N., \& Basha, S. 2017. Torque expression in self-ligating orthodontic brackets and conventionally ligated brackets: A systematic review. Journal of Clinical and Experimental Dentistry, 9(1), e123-128.

Vilella, O. V. 2007. O desenvolvimento da Ortodontia no Brasil e no mundo. Revista Dental Press de Ortodontia e Ortopedia Facial, 12(6), 131-156. 


\section{Minicurrículo}

Júlia Jordão. Acadêmica do curso de Odontologia pela Universidade São Francisco, no campus de Bragança Paulista, São Paulo, Brasil

Marcos Valério Ferrari. Graduado em Odontologia pelo Centro Universitário Herminio Ometto de Araras no ano de 1996 e especialização em Ortodontia e Ortopedia Funcional dos Maxilares pela Universidade de Guarulhos em 2001. Atualmente é Professor assistente da Universidade São Francisco. Tem experiência na área de Odontologia, com ênfase em Ortodontia.

Valdinéia Maria Tognetti. Graduada em Odontologia pela Universidade São Francisco em 2003, Especialista em Odontopediatria pela São Leopoldo Mandic em 2009, Mestre em Saúde Coletiva em Odontologia na UNICAMP/FOP em 2012, Doutoranda em odontologia com ênfase em Odontopediatria na UNICAMP/FOP (em andamento). Docente na Universidade São Francisco de Bragança Paulista no curso de Odontologia, em disciplinas teóricas, laboratoriais e práticas clínicas. Possui consultório onde é realizado clínica geral e odontopediatria. Experiência na área de Odontologia, com ênfase em Odontopediatria.

Como citar: Jordão, J., Ferrari, M.V., \& Tognetti, V.M. 2021. Eficiência de braquetes convencionais e autoligados considerando seu atrito e expressão de torque durante o tratamento. Pubsaúde, 6, a171. DOI: https://dx.doi.org/10.31533/pubsaude6.a171

Recebido: 28 abr. 2021.

Revisado e aceito: 19 mai. 2021.

Conflito de interesse: os autores declaram, em relação aos produtos e companhias descritos nesse artigo, não ter interesses associativos, comerciais, de propriedade ou financeiros que representem conflito de interesse.

Licenciamento: Este artigo é publicado na modalidade Acesso Aberto sob a licença Creative Commons Atribuição 4.0 (CC-BY 4.0). 